\title{
Traiettorie del filologicamente corretto. Il campo del folklore nell'operato di un maestro contemporaneo delle danze sarde
}

Trajectories of the Philologically Correct. The Folklore Field in the Work of a Contemporary Master of Sardinian Dances

\section{Alessandro Deiana}

\section{OpenEdition}

\section{Journals}

Edizione digitale

URL: http://journals.openedition.org/aam/381

DOI: 10.4000/aam.381

ISSN: 2038-3215

\section{Editore}

Dipartimento Culture e Società - Università di Palermo

Notizia bibliografica digitale

Alessandro Deiana, « Traiettorie del filologicamente corretto. II campo del folklore nell'operato di un maestro contemporaneo delle danze sarde », Archivio antropologico mediterraneo [Online], Anno XXI, n. 20 (1) | 2018, online dal 30 juin 2018, consultato il 28 décembre 2019. URL : http://

journals.openedition.org/aam/381; DOI : 10.4000/aam.381

Questo documento è stato generato automaticamente il 28 dicembre 2019.

Archivio antropologico mediterraneo 


\title{
Traiettorie del filologicamente corretto. Il campo del folklore nell'operato di un maestro contemporaneo delle danze sarde
}

\author{
Trajectories of the Philologically Correct. The Folklore Field in the Work of a \\ Contemporary Master of Sardinian Dances
}

Alessandro Deiana

Lectio difficilior potior ${ }^{1}$

\section{Introduzione}

1 In questo articolo ${ }^{2}$ mi propongo di mostrare alcune articolazioni poco esplorate della categoria di filologicamente corretto: ritengo che esso non sia solo uno strumento critico al servizio della visione istituzionale e colta, o uno strumento retorico a disposizione delle politiche patrimoniali: può invece anche essere impiegato dal basso ai fini di una micro-contro-egemonia locale. Il contesto culturale ed etnografico al centro di questo contributo è quella che chiamo la scena sarda del ballo "etnico", detto anche tradizionale o popolare, caratterizzato, come in generale tutto il campo del folklore contemporaneo, da un alto grado di riflessività, di tematizzazione discorsiva, nonché di messa in valore, della tradizione che si esprime, si articola e crea effetti sociali attraverso concetti e operatori retorici solitamente di provenienza colta: affianco a parole come cultura, tradizione, identità e patrimonio, gli attori di questa scena entrano ed escono da un universo retorico e sentimentale che ruota intorno alla forza insieme evocativa e performativa dell'autenticità. Tuttavia, sorge subito un problema: nel campo del folklore sardo tutti si richiamano all'autenticità - pur chiamandola non sempre con questo nome - e tutti fondano il loro operato sulla preservazione dell'autentica cultura sarda, eppure ciò non impedisce che esistano delle nette divergenze per quanto riguarda il modo di intendere l'autenticità e soprattutto 
non impedisce che si formino posizioni che non solo contestano il fatto che un certo ballo possa essere autentico, ma anche che affermano che l'autenticità di un ballo, qualora si smarrisca, deve essere accuratamente restituita attraverso la ricostruzione della sua forma originaria o più antica.

Impiegherò la categoria di filologicamente corretto per distinguere e spiegare una posizione che, a mio avviso, non può essere colta col solo concetto dell'autenticità: nel campo del folklore sardo, e in particolare nella scena della danza in quanto oggetto di questa ricerca, tutti si richiamano all'autenticità, ma solo una parte porta avanti un discorso e delle pratiche che ritengo informate al filologicamente corretto. Nell'intento di riproporre le "vere" danze del passato, in contrapposizione alla semplice evocazione retorica dell'autenticità o della purezza, questa posizione all'interno del campo del folklore sembra indicare un passaggio dalla ricerca dell'autenticità alla ricerca della verità, la quale assume spesso $i$ contorni di una lotta, considerato il conflitto tra $i$ differenti punti di vista e lo sforzo mirante ad affermare la propria scuola di pensiero.

Svilupperò la mia trattazione partendo da un inquadramento concettuale del filologicamente corretto in ambito antropologico e confrontandolo col concetto affine ma differente di autenticità. Sarà poi necessario illustrare la nozione di campo del folklore come ciò che dà senso e ragione ai processi di autenticazione e alla pratica del filologicamente corretto. Infine mi concentrerò sulla particolare traiettoria del filologicamente corretto nel contesto della pratica delle danze sarde dette tradizionali, con particolare riguardo all'operato di un maestro di ballo sardo campidanese, che tratterò alla stregua di un filologo popolare e che si muove in una scena sfaccettata della quale fanno parte gruppi folklorici, cultori della danza e ballerini di piazza. Lungo questo tracciato mi affaccerò su una serie di questioni più ampie che ho scelto di affrontare da un punto di vista specifico quale è quello della pratica e dei discorsi inerenti il ballo sardo.

Queste questioni più ampie, che attraverseranno tutto l'articolo, e che sono fronti di ricerca e versanti problematici in cui si colloca la ricerca qui esemplificata, possiamo così sintetizzarle: il rapporto tra riflessività e tradizioni popolari, ossia la questione della cultura popolare contemporanea come oggetto di discorso da parte dei soggetti popolari; le indigenizzazioni e le transculturazioni, ovvero gli usi locali e dal basso di categorie e discorsi colti e globali; la polemologia delle pratiche culturali popolari, cioè il loro essere caratterizzate - non esclusivamente, ma in misura importante - dal conflitto, dalla lotta e da movimenti tattici. Prima di tutto però illustrerò il contesto della ricerca, inteso sia come paesaggio coreutico sardo contemporaneo storicamente e culturalmente connotato, sia come modalità etnografiche che hanno orientato il mio lavoro all'interno di questo paesaggio.

\section{La scena del ballo sardo oggi come contesto di ricerca}

5 Nell'ultimo secolo anche le pratiche coreutiche sarde dette tradizionali ${ }^{3}$, come tante espressioni culturali di ascendenza popolare "pre-moderna", hanno subito un processo di oggettivazione culturale per cui fenomeni culturali complessi sono diventati oggetti culturali dal forte valore identitario per mezzo di meccanismi e apparati che li hanno naturalizzati e fissati, oggettivati, appunto (Handler 1988). Di pari passo e in relazione a 
questo processo si è anche assistito a una tendenziale e generale disaffezione, sebbene non uniforme, nei confronti del ballo sardo, scalzato o emarginato da altre forme di danza, di musica $o$, in generale, di espressività festiva ed estetica di variegata provenienza (Gallini 1971; Orrù 1999). Ciò nonostante la Sardegna rimane, secondo alcuni studiosi, «in assoluto il giacimento più ricco di balli etnici ancora praticati» nello Stato italiano (Gala 2000: 19). Ci troviamo così di fronte allo stesso tempo a una resistenza o conservatività culturale e a un proliferare inedito di pratiche e di discorsi riconducibili a una "reflexive consciousness of tradition" (Linnekin 1983: 249) che arricchiscono, anziché impoverire, il ventaglio dei problemi e degli oggetti della ricerca. In questo contesto il mio interesse è rivolto più ai danzatori e alle danzatrici in quanto attori e attrici sociali e alla loro agentività all'interno di un campo di forze che li precede e meno alla danza intesa come oggetto autonomo da analizzare da un punto di vista formale, strutturale e tassonomico.

Consapevole della vastità e della varietà del paesaggio coreutico sardo e focalizzandomi sulle pratiche e i discorsi di specifici ballerini in specifici contesti, ho condotto la mia ricerca affianco di singole persone e piccoli gruppi che, in aree geografiche e culturali circoscritte (il Basso Campidano o Campidano di Cagliari), interpretano e concepiscono il ballo sardo in un modo peculiare che è riconoscibile solo se, a sua volta, si conoscono le principali dinamiche e correnti che agiscono all'interno della più ampia scena del ballo sardo, sia da un punto di vista della trasformazione nel tempo storico, sia da un punto di vista dell'attualità. Per questo la mia ricerca si colloca sullo sfondo di una scena sfaccettata che attraverso da tempo e nella quale ho maturato una serie di relazioni e di interessi che mi fanno apparire agli occhi dei miei interlocutori a volte come amico o compagno di ballo, a volte come antropologo e altre volte tutte e due le cose assieme.

7 Dal momento che non è l'obiettivo di questo saggio fornire uno stato dell'arte sul sistema coreutico sardo e il suo studio ${ }^{4}$, penso sia sufficiente evidenziare alcuni elementi del paesaggio coreutico sardo odierno di modo da avere uno sfondo sui cui articolare $\mathrm{i}$ temi affrontati in questo articolo e contestualizzare la mia ricerca etnografica. Mi sembra che gli assi intorno ai quali si può disegnare una mappa, parziale ma anche realistica, del ballo sardo siano i tipi di esecuzione e i tipi di trasmissione della danza ${ }^{5}$. Questi elementi sono quelli che ci introdurranno all'ambito di oggetti problematici affrontati in queste pagine. Per tipi di esecuzione intendo il contesto in cui si pratica la danza e gli aspetti formali di questa pratica in base al contesto. Il tipo di trasmissione riguarda invece i modi di insegnare e di imparare la danza. Tra il tipo di esecuzione e il tipo di trasmissione c'è spesso un rapporto di corrispondenza, anche se non sempre, pertanto possono anche darsi rapporti diversi e diverse soluzioni.

8 Esistono almeno tre forme del rapporto trasmissione-esecuzione, con possibili varianti al loro interno:

1. Trasmissione empirica della danza in ambito familiare, parentale, amicale all'interno di una comunità paesana dove l'esecuzione corrispondente del ballo sardo è partecipata e collettiva in un contesto rappresentato dalle feste pubbliche (normalmente le feste dei santi e il carnevale) e private (feste familiari o di gruppi amicali: matrimoni, anniversari, feste di laurea ecc.). Questa pratica partecipata sostenuta da una competenza diffusa di lunga data coincide con una forma del ballare che, se è sbagliato definire pura, autentica o genuina, costituisce però il modello storico su cui si è fondato qualunque discorso e qualunque pratica intorno alla danza in Sardegna, compresi quelli degli studiosi. Questa situazione è tipica di 
un contesto "tradizionale" che in molte aree della Sardegna non è più operante, ma in altrettante aree è ancora oggi vivo ed esperibile. C'è chi a questo riguardo ha proposto la categoria etnocoreologica di «partecipatory dance» (Nahachewsky 1995).

2. Trasmissione formalizzata attraverso le associazioni folkloriche locali che si sono date il compito di tramandare le danze del proprio paese e spesso anche di altre aree. Questo obiettivo sfocia normalmente nella realizzazione di spettacoli ed esibizioni in costume sia nel proprio paese che in altre località, in svariati contesti, che a volte esulano da quelli "tradizionali". In questo caso il tipo di pratica della danza corrispondente è appunto la pratica della danza sul palco o "presentational dance» (Nahachewsky 1995). Molte volte dove opera questa forma di trasmissione e di esecuzione non è più presente il ballo eseguito collettivamente in piazza. Ma dato che in Sardegna quasi ogni paese ha almeno un gruppo folklorico, questa situazione può presentarsi anche in paesi dove il ballo è ancora praticato in piazza, pertanto possiamo trovarci di fronte a una situazione in cui convivono trasmissione empirica e formalizzata, pratica partecipatoria e presentazionale. Tuttavia, dove il primo tipo di rapporto trasmissione-esecuzione è ancora vitale e diffuso, questo è in grado di agire sul secondo tipo, nel senso che chi fa parte del gruppo folklorico paesano può anche aver imparato il ballo per via empirica e qualunque componente può anche praticare il ballo in piazza nel proprio paese, oppure in altri paesi o in altri contesti coreutici. In questo tipo di esecuzione del ballo sardo il modello storico costituito dal ballo in piazza può essere rappresentato in base a ciò che definisco un arco rappresentativo che va dalla riproducibilità fedele (ovvero la rappresentazione sul palco, nei limiti dei condizionamenti spettacolari, di quel modello secondo criteri che possiamo chiamare filologici, come vedremo meglio più avanti) alla interpretabilità creativa (ossia la rappresentazione di quello stesso modello sfruttando in maniera innovativa le caratteristiche della forma-spettacolo: rapporto con gli spettatori, tempi, coreografie ecc.) (Deiana 2017: 206-213).

3. Trasmissione formalizzata attraverso corsi di ballo sardo specifici, il cui obiettivo è formare nuovi ballerini e ballerine che possano eseguire danze in piazza, quindi in un contesto partecipativo o "tradizionale" e non sul palco, cioè in un contesto presentazionale o spettacolare, come invece fanno i gruppi folklorici. Normalmente si iscrivono e partecipano a questi corsi persone di tutte le età che non hanno mai ballato il ballo sardo e vorrebbero impararlo. Possono capitare anche persone che sanno già ballare (magari le danze del proprio paese), ma vorrebbero imparare balli che ancora non conoscono (quelli di altri paesi o altre regioni della Sardegna): in questo caso possono essere persone che hanno una competenza maturata o in un contesto del primo tipo o del secondo tipo. Anche gli insegnanti possono avere una diversa formazione: c'è chi ha imparato a ballare empiricamente da bambino in un contesto partecipativo con alti livelli di competenza diffusa, c'è chi ha imparato all'interno di gruppi folkloristici e poi magari perfezionandosi con ricerche personali, c'è anche chi ha imparato in età più tarda al seguito di un maestro elettivo, prima, dopo, nel mentre o al di fuori di qualche esperienza in gruppi folklorici. Quello dei corsi di ballo sardo, spesso chiamate «scuole di ballo sardo», sono un fenomeno recente che inizia grosso modo nei primi anni Duemila, diffuso specialmente a Cagliari e nel suo circondario (Calzia 2014: 97) e che opera una congiunzione tra la trasmissione formalizzata dei gruppi folklorici e la pratica di piazza dei contesti detti tradizionali ${ }^{6}$. Qui la tendenza è quella di riproporre il modello storico secondo il principio di riproducibilità fedele perché è quello che secondo insegnanti e allievi va eseguito nella pratica di piazza a cui questi corsi intendono preparare. Tuttavia, il tipo di trasmissione formalizzata a cui solitamente le scuole si affidano a volte può risentire di stilemi e forme coreografiche tipiche dell'interpretabilità creativa di alcuni gruppi folklorici.

9 Concludo questi schizzi del paesaggio coreutico sardo, che sono stati fin qui di ordine sincronico, con un'annotazione da un punto di vista diacronico. Questo riferimento è importante perché ci aiuta a capire ulteriormente il contesto in cui intendo utilizzare la 
categoria del filologicamente corretto. Mi ricollego all'asse trasmissione-esecuzione e alle sue tipologie. La prima è contrassegnata dalla continuità storica, da intendere come una continuità documentata della pratica collettiva e partecipata del ballo sardo in contesti festivi in singoli paesi. Questa continuità non significa ovviamente un'immutabilità astorica delle forme concrete del ballare, ma semmai un'evoluzione storicamente determinata all'interno di una relativa stabilità della pratica e della competenza. La seconda tipologia invece può presentarsi sia in contesti di continuità (abbiamo detto che i gruppi folklorici sono presenti anche in paesi dove ancora si balla in piazza), ma anche in contesti di discontinuità, ovvero dove si è interrotta la pratica collettiva e partecipata del ballo in piazza e ad essa si è sostituita la pratica specialistica del ballo sul palco ad opera dei gruppi folklorici. La terza e ultima tipologia, che ha anche una genesi storica più recente, opera anche in questo caso una sorta di ricongiunzione tra passato e presente, cioè costituisce la ripresa di ciò che si è interrotto da tanto tempo, ma attraverso modalità di trasmissione che si distinguono da quelle empiriche e che hanno introdotto in tempi relativamente recenti i gruppi folklorici.

10 Rispetto al rapporto diacronico del ballo sardo con la storia, ci troviamo dunque di fronte a dinamiche di continuità, interruzione (o discontinuità) e ripresa. È in fondo in relazione a tutte e tre queste dinamiche storiche che agisce la categoria del filologicamente corretto. Essa può rispondere ad una serie di problemi che emergono quando il principio dell'autenticità non riesce più ad articolare in maniera credibile il rapporto trasmissione-esecuzione attraverso le forme della continuità, dell'interruzione e della ripresa delle pratiche coreutiche etnicamente connotate come sarde in un contesto storico di grandi cambiamenti culturali e in un campo specifico, quello del folklore, assai variegato e conflittuale.

\section{Filologicamente corretto e autenticità}

11 È stato probabilmente Berardino Palumbo a introdurre il tema del filologicamente corretto come categoria intellettuale che, nell'ambizione di alcuni soggetti di sottoporre a un giudizio critico informato a valori scientifici ed estetici di provenienza colta e istituzionale cose e azioni che sfidavano tali valori, ha lasciato in ombra sia tutto il mondo sociale che genera e riproduce tali oggetti e comportamenti filologicamente scorretti, sia la genesi storica e le implicazioni politiche di questa categoria e del suo uso istituzionale e non (Palumbo 2003). Palumbo ha invitato a rivolgere uno sguardo antropologicamente critico su una nozione che da una parte costituisce una risorsa intellettuale esplicativa nei confronti di una serie di fenomeni culturali e dall'altra è da considerarsi invece una pratica discorsiva che richiede di essere spiegata e decostruita a sua volta, sia dal lato di soggetti colti e istituzionali che si avvalgono di questa categoria in maniera quasi monopolistica, sia dal lato di variegati soggetti locali e popolari che con questa categoria hanno un rapporto molto più complesso e articolato di quanto i punti di vista istituzionalmente legittimati sono in grado di vedere.

Palumbo introduce il tema del filologicamente corretto nella sua etnografia sui processi di patrimonializzazione e di oggettivazione culturale in Sicilia sud-orientale. Nel suo caso questi processi riguardano il patrimonio culturale storico-artistico-religioso, ma li studia da un punto di vista da cui è possibile osservare il complesso campo simbolicopolitico che si forma alla confluenza di multiformi istanze di natura locale e globale, 
popolare ed elitaria, colta e vernacolare, naif e istituzionale. È questa prospettiva che ha permesso a Palumbo di decostruire il discorso colto e ufficiale sul patrimonio culturale, ossia quello improntato al paradigma del filologicamente corretto, e di mettere in luce, allo stesso tempo, la contestazione pratica di quel discorso a livello locale.

Ma che cosa è più precisamente il filologicamente corretto? Che cosa si vuole dire con questa forma aggettivale che rimanda a una disciplina come la filologia e a una correttezza che questa disciplina attribuisce o invalida? Innanzitutto va detto che né Palumbo né io ci riferiamo al corpus teorico e metodologico proprio della filologia, ma semmai all'evocazione della correttezza filologica fatta da diversi soggetti, peraltro quasi mai filologi propriamente detti, in diversi contesti e con diverse finalità. Si tratta quindi di un uso multiforme del concetto di correttezza filologica che spesso eccede il campo della filologia per svilupparsi in territori simbolici che sono più frequentati dall'antropologia culturale e meno, credo, dalle discipline filologiche. Ma sopratutto lo stesso Palumbo non definisce questa nozione e non la teorizza esplicitamente, anche perché non è l'oggetto centrale della sua ricerca, ma ne lascia emergere il significato nel corso della sua trattazione tutte le volte che usa questo concetto per mostrare il gioco di frizioni, spesso stridenti, tra le rappresentazioni ufficiali del patrimonio culturale storico-artistico improntate a valori strettamente connessi alla storia degli Stati-nazione e gli usi locali dello stesso patrimonio che agiscono secondo altre logiche culturali, ma non per questo meno politiche e meno immerse in relazioni di potere. Che cosa sia il filologicamente corretto diventa allora molto chiaro quando Palumbo mette a confronto regimi di verità differenti di fronte a determinati usi di beni culturali: pratiche devozionali e costruzioni dell'appartenenza collettiva che prendono la forma di addobbi "pacchiani" su altari e simulacri barocchi, da una parte, e l'immaginario estetico e patrimoniale che prende corpo nell'indignazione dello storico dell'arte per questi "scempi", dall'altra (Palumbo 2003: 17-25, 39-41).

Tolto il contributo di Palumbo, non sembra esistere una letteratura antropologica sul filologicamente corretto o, meglio, sull'uso della nozione di filologicamente corretto in quegli ambiti e all'interno di quelle problematiche che sono soliti attraversare gli antropologi. Nemmeno riguardo a quei campi di ricerca in cui Palumbo la impiega e che sono ormai da anni tra quelli privilegiati dalla ricerca antropologica contemporanea, ossia i processi di patrimonializzazione ${ }^{7}$. L'unico riferimento al filologicamente corretto come nozione specifica dell'antropologia dei patrimoni lo si può trovare in un articolo di Chiara Bortolotto, dove a questo riguardo viene citato Palumbo e la "philological correctness» viene accostata alla «search for authenticity» (Bortolotto 2007: 27) ${ }^{8}$. Il filologicamente corretto e l'autenticità sembrano essere due cose che si richiamano a vicenda, specialmente dal lato colto e ufficiale dei processi di patrimonializzazione. Bortolotto infatti si riferisce al concetto di autenticità come parola chiave che per molto tempo ha ispirato il paradigma patrimoniale «based on philologically determined authenticity» (Ibidem), a partire dalle convenzioni ufficiali dell'Unesco sulla salvaguardia del patrimonio immateriale dell'umanità fino alle singole candidature di un determinato patrimonio di un determinato paese per l'iscrizione alle liste Unesco. Qui per autenticità si intende il vero che si oppone al falso o l'originale che si oppone alla copia, ma anche il genuino al contaminato, lo spontaneo all'artefatto, il radicato nel passato all'inventato nel presente. Benché la categoria di autenticità sia stata espunta dalle più recenti convenzioni Unesco, proprio in seguito a un ripensamento critico delle suddette opposizioni binarie, essa rimane ancora centrale nel discorso e nelle pratiche 
patrimoniali e per tutti i soggetti coinvolti (Bortolotto 2013). L'autenticità, dunque, sembra ancora essere una categoria irrinunciabile in qualunque situazione di tematizzazione riflessiva e di oggettivazione della cultura quali sono il patrimonialismo e i processi di patrimonializzazione. Tuttavia, l'autentico e il filologicamente corretto mantengono la loro rispettiva autonomia: l'autenticità è un portato del pensiero e del sentimento occidentale moderno (Bendix 1997; Lindholm 2013) dal quale la filologia dipende, ma la filologia è lo strumento principale per affermare l'autenticità nella misura in cui essa corrisponde al filologicamente corretto.

Oltre a questo, autenticità e filologicamente corretto esprimono, da parte dei soggetti in campo, due diverse declinazioni di quel regime di riflessività culturale che caratterizza non solo il patrimonialismo ufficiale, ma anche il più vasto folklore contemporaneo, nella fattispecie quello sardo, dove l'idea di patrimonio culturale agisce indipendentemente dal paradigma dell'Unesco e si sostanzia in pratiche e discorsi che si muovono creativamente tra ballerini e suonatori, gruppi folklorici, studiosi locali, assessorati alla cultura, uffici regionali, scuole di danza, singoli appassionati, emittenti locali ed eventi pubblici. In questo contesto, così come avviene nella scena del ballo sardo nel Basso Campidano, alcuni soggetti individuali e collettivi non solo esprimono un'autocoscienza culturale, tematizzano esplicitamente il valore della tradizione e rivendicano l'autenticità delle loro riproposizioni folkloriche - e ciò accomuna tutti gli attori della scena - ma, a differenza di altri, vogliono riproporre la tradizione come era veramente in passato, cioè in maniera filologicamente corretta: in questo caso la categoria dell'autenticità si presenta con maglie troppo larghe per catturare un fatto che sembra non aderire più al modello della ricerca dell'autenticità, ma semmai a quello della ricerca della verità.

\section{Filologicamente corretto e campo del folklore}

Durante una visita alla sede di un gruppo folklorico del cagliaritano, un ballerino e dirigente mi spiegò la struttura e il significato di una nuova coreografia creata dal suo gruppo: nonostante questa coreografia avesse molto poco di filologicamente corretto (addirittura vedeva la partecipazione di una «ballerina di danza classica»), alla fine della sua descrizione ci tenne a precisare che: «noi facciamo folklore puro». La maggioranza spettacolarista o modernista o innovatrice della scena campidanese - e di cui questo mio interlocutore fa parte - si guarda bene dall'esplicitare troppo nettamente la sua posizione oggettiva, avendo cura invece di rivendicare la sua appartenenza legittima alla scena evocando l'autenticità della tradizione che presenta nelle sue performance. L'evocazione dell'autenticità da parte dei gruppi folklorici agisce come il richiamo alle genealogie di lignaggio da parte degli abitanti delle oasi tunisine studiate da Mondher Kilani: funziona da supporto retorico alle strategie di legittimazione all'interno del contesto locale, poiché dispiega degli effetti di persuasione utili a istituire e gestire relazioni sociali (Kilani 1992). Il richiamo alla purezza, in fondo, ha a che fare più con i processi di autenticazione che con l'autenticità (de Sanctis Ricciardone 2007: 138-139). Queste strategie retoriche e i processi di autenticazione a cui sono sottese trovano il loro senso e la loro ragione all'interno di quello che chiamo campo del folklore ${ }^{9}$. Definisco campo, sulla scia di Pierre Bourdieu, quello spazio materiale e simbolico insieme che viene proiettato dalle azioni dei soggetti che sono coinvolti in esso a partire dallo specifico presupposto indiscusso 
che ne motiva l'agire e che dunque fonda il campo stesso (Bourdieu, Wacquant 1992; Bourdieu 1998).

Il campo costituisce così un microcosmo relativamente autonomo capace di esercitare la sua forza sui soggetti in esso e per esso coinvolti. Ora, il presupposto che fonda in generale il campo del folklore e in particolare quello del ballo sardo è la credenza nel valore in sé delle tradizioni sarde e nella necessità di preservarle nella loro autenticità. Questa credenza è ciò che Bourdieu chiamerebbe l'illusio del campo. L'illusio (dal latino 'ludere', giocare, essere nel gioco) è il presupposto di partenza da cui si costruisce un campo specifico; è la credenza, la fede o la convinzione a cui i giocatori devono aderire di modo che il gioco sia preso come una cosa seria e di cui ne valga la pena; l'illusio è la matrice a partire dalla quale si stabilisce ciò che è importante e ciò che non lo è in un dato campo. L'illusio del campo del folklore è ovviamente il folklore, per la precisione è il valore fondante e indiscusso del folklore e delle tradizioni in quanto tali, l'importanza di affermarne l'esistenza e di preservarli. L'illusio del folklore costituisce allo stesso tempo l'indiscusso fondamento simbolico che dà senso al campo e ciò che fornisce una serie di operatori retorici che permettono la manipolazione di memorie, storie, identità in vista del raggiungimento di determinati interessi e di stabilire posizioni (Faubion 1993): queste ultime dipendono infatti dal diverso grado di capitale (economico, politico, culturale e sociale) detenuto dai soggetti individuali e collettivi che aderiscono all'illusio del campo del folklore, nonché dalle relazioni che si creano tra di loro e dalle traiettorie storiche di ogni soggetto. La metafora della traiettoria va quindi intesa non come un movimento lineare che disegna una continuità tra il punto di partenza e quello di arrivo, ma come una serie discontinua di spostamenti nello spazio sociale dipendenti dalle relazioni che si definiscono con altre posizioni e altri soggetti (cfr. Bourdieu 1995: 67-68). Le traiettorie del filologicamente corretto dipendono allora dai soggetti che le incarnano a partire da determinate posizioni e in determinati campi, non di rado confinanti (accademico, politico, patrimoniale, folklorico ecc.).

Il campo del folklore sardo a sua volta comprende dei sotto-campi che possiamo individuare in quegli spazi pertinenti che ruotano intorno a una singola determinata espressione culturale che nel campo del folklore è riconosciuta come uno degli elementi costitutivi della credenza nel valore delle tradizioni popolari: così possiamo avere un sotto-campo della musica, del canto, del vestiario, della poesia e, quello che qui interessa a me, della danza. Un primo approccio al sotto-campo del ballo sardo oggi è in fondo quello che ho precedentemente illustrato in base alla relazione tra trasmissione ed esecuzione sul piano sincronico e tra continuità e discontinuità sul piano diacronico. In questo avvicinamento al sotto-campo della danza abbiamo iniziato a vedere alcuni soggetti che lo costituiscono in qualità di coloro che coprono delle determinate posizioni e perseguono dei determinati obiettivi, ovvero gli esecutori del ballo in piazza (sia nella veste dei continuatori della tradizione coreutica del proprio paese che in quella di chi, attraverso le scuole di ballo, ha scoperto la pratica della danza e la insegue attraverso le feste in lungo e in largo per la Sardegna, spesso venendo dalle città dove il ballo in piazza si è estinto) e gli esecutori specializzati del ballo sul palco, cioè i gruppi folklorici.

Per completare questo quadro d'insieme dei principali soggetti in campo vanno ricordati i singoli cultori e a volte maestri di ballo intorno ai quali spesso ruota un corso o una scuola di ballo: queste figure associano alla pratica del ballo un approfondimento di natura storica o antropologica all'oggetto della loro passione e del loro interesse ${ }^{10}$. 
Tenendo presente che questi soggetti possono anche sovrapporsi, nel senso, per esempio, che un maestro di ballo può anche appartenere a un gruppo folklorico e praticare il ballo di piazza, tenendo dunque presente questi incroci pratici bisogna inoltre considerare la distribuzione dei diversi tipi di capitale e le diverse disposizioni ideologiche che definiscono le posizioni in campo dei soggetti e le loro traiettorie disegnate dal perseguimento di interessi e dalla ricerca di "profitti". Nell'area del Basso Campidano che sto prendendo in esame i gruppi folklorici sono indubbiamente quei soggetti, in particolare nella persona dei loro dirigenti, col più alto grado di capitale politico e sociale (pur con delle differenze da un gruppo a un altro). Ovvero, sono quei soggetti storicamente legittimati a riproporre le tradizioni del proprio paese.

Per legittimazione storica intendo che i gruppi folklorici sono il prodotto conseguente di un processo storico oggettivo di rarefazione delle tradizioni coreutiche sarde e allo stesso tempo di salvaguardia di queste da parte di minoranze specializzate: questa legittimazione in molti casi, anche se non in tutti, si presenta come forma di delega, se non collettiva perlomeno istituzionale o politica, a rappresentare le tradizioni della Sardegna e del proprio paese ${ }^{11}$. Ciò fa in effetti di queste minoranze dei soggetti che rappresentano un notevole consenso di massa: basti vedere il pubblico che accorre ai loro spettacoli. Tale legittimazione in quest'area della Sardegna è rafforzata dal fatto che i gruppi folklorici, per via della scomparsa della pratica collettiva del ballo in piazza, posseggono una sorta di monopolio del ballo. Questa peculiare posizione dei gruppi folklorici fa di loro anche i primi destinatari di quelle risorse pubbliche riservate al folklore organizzato, o perlomeno i più attrezzati ad attingerle ${ }^{12}$. Sono i gruppi folklorici infatti a rappresentare la tradizione nelle principali ricorrenze festive: dalle più imponenti feste dell'Isola alla festa patronale del più piccolo dei paesi.

21 Le scuole di ballo, da questa prospettiva, ricoprono invece una posizione subordinata, anche da un punto di vista numerico, poiché al massimo vengono coinvolte ogni tanto in qualche evento per animare la piazza con i loro ballerini. Questi ultimi, in area campidanese, non costituiscono più un soggetto collettivo in grado di determinare le sorti del ballo dal momento che la competenza coreutica è ridotta a sparute e sparse minoranze che, in quanto tali, non sono in grado di riprodurre, se non in alcuni casi, la pratica comunitaria del ballo di piazza. Tuttavia, alcuni ballerini e cultori del ballo sardo rappresentano un ulteriore posizionamento nella scena locale in virtù soprattutto di una divisione ideologica operante nel sotto-campo della danza, cioè quella tra i fautori dell'introduzione della forma-spettacolo e i fautori del rigore filologico. La maggioranza schiacciante della scena è schierata con i primi, ma la semplice esistenza di questa contrapposizione mette in luce la minoranza dei filologici e ne mostra chiaramente la sua posizione in campo.

22 I processi di autenticazione che contribuiscono a creare il campo del folklore alla lunga hanno dato esito, da una parte, a delle interpretazioni creative della tradizione e, dall'altra, a delle reazioni ad esse di ordine filologico, ovvero dei tentativi di ristabilire il vero testo coreutico andato perduto nonostante $i$, o proprio a causa dei, processi di autenticazione propri del campo del folklore del Sud Sardegna. Qui il filologicamente corretto entra in gioco sotto una nuova veste quando il principio dell'autenticità viene richiamato discorsivamente dalla frazione egemone della scena locale, ma di fatto non concretizzato nella performance coreutica. Il filologicamente corretto entra in gioco anche come meta-concetto che ci consente di affacciarci su tutti e due i versanti che esso ricopre, ma che difficilmente, prima dell'uso che ne ha fatto Palumbo, apparivano 
illuminati contemporaneamente: quello dei soggetti definitori della correttezza filologica e quello dei soggetti che diventavano oggetto di tali definizioni, e che a loro volta presero ad avvalersi delle definizioni dei definitori. Si tratta dello scambio e del passaggio di consegna del filologicamente corretto dalla dimensione colta degli studi di antropologia e di folklore classici alla dimensione «vernacolare» (Bauman 2008) dei filologi popolari. O, per dirla con Gramsci, del filologicamente corretto come una di quelle «nozioni scientifiche» che «più o meno sfigurate, cadono continuamente nel dominio popolare» (Gramsci 1975: 2312).

\section{Dagli antropologi ai nativi: filologia popolare}

Un altro elemento, dunque, che contribuisce a delineare il campo del folklore nella Sardegna meridionale, oltre a una riflessività diffusa (intesa come il dispiegamento di un discorso esplicito sulla propria soggettività individuale e collettiva in quanto legata a un'identità culturale e a un corpus di tradizioni oggettivate), lo possiamo richiamare con le parole con cui la demologia ha definito il passaggio di un'espressione culturale dall'alto al basso della gerarchia sociale, ovvero un ormai consolidato "processo di discesa»: nella scena locale del folklore riflessivo infatti non si può non fare ampio uso di nozioni e idee di provenienza colta, globale o egemone. Ma a discendere sono state anche le stesse categorie che gli antropologi impiegavano per studiare le culture subalterne e i dislivelli di cultura: non solo quindi «fatti culturali» generalmente provenienti dalle classi dominanti e fatti propri dai dominati, ma «nozioni scientifiche» che per molto tempo avevano visto i subalterni come oggetto di conoscenza e che ora, da qualche tempo a questa parte, li vede come soggetti che maneggiano tali nozioni per parlare di sé stessi e per portare avanti determinate idee e determinate pratiche.

Ci troviamo così di fronte non solo a forme di indigenizzazione di espressioni culturali provenienti dal centro dell'ecumene globale (Appadurai 2001; Hannerz 2001), ma anche a processi di transculturazione nel senso in cui li ha intesi Mary Louise Pratt, ovvero come il modo in cui «subordinated or marginal groups select and invent from materials transmitted to them by a dominant or metropolitan culture» (Pratt 1992: 6). Negli studi sul folklorismo questo fenomeno fu già messo in luce negli anni ' 60 del secolo scorso da Hans Moser nei termini di un feedback (Rücklauf) che si manifesta quando i portatori delle tradizioni incorporano concetti scientifici o pseudo-scientifici nelle loro pratiche discorsive inerenti il folklore (Bendix 1988: 6). Concetti di origine colta, e fondanti per l'antropologia, come cultura, tradizione, identità, differenza, folklore, etnia e patrimonio sono indispensabili nel discorso del folklore riflessivo contemporaneo. $\mathrm{E}$ qua e là, nel corso della ricerca, mi è capitato perfino di sentire pronunciare termini specialistici come «rituale», «matriarcato», «apotropaico» ecc. Ma oggi è addirittura un metodo o una postura conoscitiva ad essere discesa riflessivamente dagli studi accademici alla cultura popolare: mi riferisco a quell'approccio filologico che per molto tempo ha contraddistinto il modo di rapportarsi degli antropologi nei confronti del folklore revivalista, ovvero la verifica della correttezza storica ed etnografica delle espressioni culturali rappresentate (Deiana 2013; Mirizzi 2007; Palombini 2007).

L'interesse filologico, oltre che tassonomico, ha dominato per molto tempo gli studi di folklore che si sono occupati in particolar modo di letteratura orale popolare: stabilire il testo archetipico e ricostruire la serie di varianti prodotte dalla tradizione. Preoccupazione che non è cambiata quando si è trattato di confrontarsi con un 
fenomeno nuovo: il cosiddetto folklorismo. Anche se questo appariva in un periodo storico in cui iniziava ad affermarsi negli studi italiani quella che Alberto Cirese ha chiamato «la nuova tematica socio-culturale» di ispirazione gramsciana (Cirese 1973: 194), gli antropologi valutavano da una posizione filologicamente critica il «secondhand folklore» (Bendix 1988: 6): la riproposizione di un testo culturale (sia esso un canto, una musica, una danza, un rituale) doveva avvenire nella maniera più rispettosa possibile dei criteri storiografici ed etnografici necessari alla sua ricostruzione, ovvero doveva essere filologicamente corretto.

Questa preoccupazione degli antropologi in fondo ci dice che spesso e volentieri i gruppi e le associazioni di riproposta folklorica non rispettano questi criteri. L'incontro-scontro tra i regimi di verità degli antropologi e del folklore riflessivo contemporaneo (Deiana 2012) ha molto in comune con l'incontro-scontro tra gli storici e i critici d'arte e gli usi e le concezioni popolari del patrimonio culturale di cui parla Palumbo. Ma ciò che mi sembra più rilevante è appunto il fatto che il principio di metodo di cui gli antropologi e i folkloristi erano gli esclusivi detentori ora è transitato presso i portatori di folklore. Della filologia, che per molto tempo è servita a rispondere al tipo di problemi che si ponevano i folkloristi e a costruire lo stesso oggetto del loro sapere, oggi se ne sono appropriati i subalterni. La filologia, che un tempo mediava il rapporto scientifico tra il folklorista e il suo oggetto, ora media il rapporto tra gli agenti popolari e la loro cultura, o meglio media le relazioni che gli attori del campo del folklore possono intrattenere tra di loro a partire da diverse posizioni.

Si è delineato così un nuovo ordine di problemi per lo studioso: ciò con cui si voleva comprendere alcuni fenomeni culturali, la filologia, ora è diventato ciò che va a sua volta compreso nella misura in cui se ne è appropriata quella parte del mondo sociale di cui solitamente si occupano gli antropologi. Ampliando e sviluppando, quindi, ciò che ci ha mostrato Palumbo del filologicamente corretto come operatore retorico dai potenti effetti performativi all'interno di relazioni sociali di potere. Il filologicamente corretto di cui parla Palumbo si irradia in particolar modo dal lato egemone di queste relazioni. Ma rimane più che mai aperto il ventaglio di possibilità su dove e come queste irradiazioni si diffondono e hanno effetto. Per questo parlerò di filologia popolare, come, per esempio, Palumbo parla di «saperi storiografici locali» (Palumbo 2003). Ciò per distinguere la filologia scientifica ed egemone da quella naif e subalterna, ovvero l'esito della discesa e dell'appropriazione della filologia colta nella dimensione locale e vernacolare.

28 Allo stesso tempo, tuttavia, l'idea di saperi filologici popolari o locali può servire a mettere in luce l'aria di famiglia che accomuna le pratiche filologiche scientifiche e quelle informali, ossia la storia di uno scambio e di un transito di saperi dal livello egemone al livello subalterno sul terreno comune del discorso sulla cultura popolare. Oltre a ciò, ritengo che portare su un piano informale e vernacolare il filologicamente corretto può costituire un contributo a riconoscere e distinguere ulteriori sfaccettature e articolazioni delle forme di vita popolari, come i micro-poteri e le micro-resistenze operanti anche a livello subalterno, dal momento che il filologicamente corretto esprime una risposta di una minoranza insoddisfatta e critica nei confronti di un discorso maggioritario ed egemone, quello dell'autenticità delle tradizioni, all'interno della scena del folklore del Basso Campidano.

È dunque sull'operato di un filologo popolare, Tonio Spiga, ballerino e maestro di ballo, che concentrerò il focus etnografico: egli si trova ad operare all'interno delle tre 
dinamiche storiche del ballo sardo precedentemente individuate (continuità, tradizione e ripresa), ma in particolare nella dimensione della ripresa, dal momento che lui da anni organizza dei corsi di ballo in un'area, il Campidano di Cagliari, dove la trasmissione e l'esecuzione del primo tipo si sono interrotte da più di mezzo secolo. Con una caratteristica che forse lo rende un unicum: i suoi corsi di ballo sono rivolti esclusivamente al ballo detto campidanese (mentre gli altri corsi propongono varietà di ballo di tutta la Sardegna) e all'insegnamento di questo ballo in tutta la sua complessità (il che, da quello che mi è dato sapere, non avviene negli altri corsi), complessità che lo rendo il ballo di più difficile esecuzione e il meno eseguito. È anche questa unicità che $\mathrm{mi}$ ha stimolato a sperimentare l'efficacia della categoria del filologicamente corretto in questo campo particolare.

\section{Un filologo popolare del ballo sardo campidanese: dalla ricerca dell'autenticità alla ricerca della verità}

30 Tonio Spiga è un danzatore di ballo sardo e maestro ${ }^{13}$ della variante bassocampidanese ${ }^{14}$, nonché suonatore di organetto e collezionista di costumi sardi d'epoca. Ha 64 anni e di professione è insegnante di storia dell'arte alle scuole medie inferiori. È nato a Quartucciu, un paese alle porte di Cagliari e là viveva fino a pochi anni fa quando si è trasferito a Quartu Sant'Elena, città confinante. La sua famiglia era di estrazione contadina: suo padre era un medio proprietario di terreni agricoli votati fondamentalmente alla produzione vitivinicola; la madre massaia. Tonio ha iniziato ad apprendere i primi rudimenti della danza da bambino. Un momento e un luogo determinante per il suo apprendistato coreutico è stato su magasinu (la bettola o la mescita) gestito dai genitori nella casa di famiglia a Quartucciu: questi luoghi erano locali alla buona adiacenti a una cantina dove gli uomini si incontravano solitamente a fine giornata per bere in compagnia, ma anche per suonare e improvvisare qualche canto. Fu lì che Tonio ebbe modo di conoscere il ballo sardo campidanese. Già allora (siamo tra gli anni ' 50 e gli anni ' 60 del secolo scorso) si trattava di uomini anziani che risultavano gli ultimi depositari di un sapere ormai ignorato o disdegnato dalle giovani generazioni: a Tonio bambino i primi passi furono insegnati proprio da questi anziani. Nato in un ambiente che comunque lo invogliava al folklore, visto che il padre faceva parte di comitati preposti all'organizzazione delle feste e che in famiglia si conservavano vecchi "costumi" che lui indossava in occasioni festive, crescendo ha preso a girare la Sardegna per migliorare le sue conoscenze coreutiche, imparando da ballerini di altri paesi e raccogliendo documenti fotografici e audiovisivi. Questa ricerca si fecce ancora più motivata quando da ragazzo, entrato a far parte di un gruppo folklorico del suo paese, si rese conto che il ballo che proponeva aveva poco a che fare con quello che lui aveva conosciuto presso gli anziani in su magasinu e in altri paesi del Campidano. La delusione per l'esperienza folkloristica lo spinse ulteriormente a ricercare e a ricostruire con la maggior cura possibile il "vero" ballo campidanese tradizionale:

Poi negli anni '70 sono entrato a far parte di un gruppo folkloristico. Mi avevano invitato a farne parte, perché comunque era una cosa che mi piaceva e ho iniziato lì a ballare. Però poi avevo notato per esempio per quanto riguarda il campidanese che non era come quello che avevo visto. Non è che prima io ballavo, però sapevo come era. Però era diverso, vedevo che era diverso. Allora ho cominciato a girare per i paesi, Burcei, tutti questi paesini qua e vedere veramente come era e avevo 
notato le differenze [...]. E a un certo punto ho abbandonato il gruppo folkloristico perché capivo che stavano facendo solo scemenze, cose che non c'entravano niente. Quando ho cominciato a conoscere i balli anche dell'interno della Sardegna, proprio i balli tradizionali, non riuscivo più a fare i balli dei gruppi folk perché capivo che era una cosa che non stava né in cielo e né in terra. E allora ho mollato anche il gruppo folk ${ }^{15}$.

31 Questo discernimento delle «differenze» tra il ballo proposto dai gruppi folklorici campidanesi e «i balli tradizionali» si fonda per Tonio sul fatto di parlare «per conoscenza di vissuto, quello che ho potuto aver visto e sentito da testimonianze di persone che le hanno vissute in prima persona» ${ }^{16}$. Si palesa così il criterio storiografico di Tonio, che si affida alle fonti vive dei testimoni e dei "superstiti" per tracciare, in una sorta di mappa storica, i limiti temporali della tradizione:

L'unico riferimento è con gli anziani di allora, degli anni ‘70, che erano i giovani del 1900 , dove conoscevano tutti il ballo tradizionale. Che poi se andiamo indietro di trecento anni fa magari era anche un po' diverso. A me non interessa sapere come era quattrocento anni fa, come era nel 1500, non lo so. Sicuramente quell'impronta ce l'aveva, l'impronta era quella. Magari aveva altre caratteristiche che i nostri nonni, bisnonni non hanno più usato. Sicuramente, perché l'evoluzione c'è sempre stata. A me interessava conoscere l'ultimo ballo popolare, l'ultimo che è stato ballato dal popolo ${ }^{17}$.

Naturalmente tutti i gruppi folklorici e tutti i cultori locali, chi più e chi meno, hanno ricercato e ricercano un rapporto conoscitivo con gli anziani depositari del sapere coreutico proprio del loro paese. Ma è esattamente su questo punto che insiste il puntiglio filologico di Tonio, ovvero sul fatto che di fronte agli ultimi testimoni viventi di una tradizione non c'è stato un interesse e un impegno che fossero all'altezza della difficoltà di apprendere il ballo campidanese in tutta la sua complessità:

È finito perché con la guerra poi ha rovinato tutto, con l'ultima guerra soprattutto poi... Sino agli anni '30 si ballava dappertutto, anche qui si ballava molto ancora. Poi con la guerra, quindi... Ecco, quando invece [...] hanno iniziato con le pro loco, con i gruppi folk negli anni ' 50 e ' 60 , lì potevano attingere bene dai loro vecchi, capito?, dai genitori, dai nonni. Invece non l'hanno fatto. Hanno...siccome hanno capito che era difficoltoso hanno preferito fare una cosa molto levigata, molto liscia liscia eh [ride] ${ }^{18}$.

33 Come già accennato, il paesaggio coreutico in cui si muove Tonio è caratterizzato da una parte dalla discontinuità storica della pratica del ballo in piazza come fenomeno collettivamente praticato e dall'altra dalla ripresa di quella pratica, attraverso le associazioni folkloriche, secondo una modalità che ha restituito il ballo campidanese in una forma semplificata, stilizzata e con l'aggiunta di nuove coreografie. Ciò non impedisce ai gruppi folklorici di sostenere l'autenticità delle danze che ripropongono, sopratutto per il fatto di appartenere a un campo, quello del folklore, che ingiunge simbolicamente ai suoi appartenenti ad aderire all'illusio del campo, ovvero al valore in sé della tradizione e della tradizione in quanto autentica. I gruppi folklorici costituiscono per Tonio i principali avversari poiché essi nei loro spettacoli rappresentano, come dice solitamente Tonio, «una cosa inventata», cioè una danza che non corrisponde a quella che veramente si eseguiva in Campidano quando ancora si praticava il ballo in piazza.

Questa caratteristica del pensiero e dell'operato di Tonio mi fa dire che essi siano informati al principio della correttezza filologica, ossia della ricostruzione, attraverso il confronto dei testi noti, del modello primario del testo coreutico che più si avvicina all'ipotetico originale o, per dirla col lessico dei filologi, del suo archetipo (Cfr. Contini 
1986: 20-21). Rispetto al principio dell'autenticità costantemente richiamato dai gruppi di ballo del Campidano, Tonio sembra infatti perseguire una ricerca della verità storica sul ballo campidanese che deve tradursi in una ricostruzione filologicamente corretta. Mentre la maggioranza della scena locale evoca un'autenticità insieme «espressiva» in quanto trasmette valori, credenze e significati (Dutton 2003): in questo caso i gruppi folklorici con le loro danze esprimono l'autentica cultura etnica dei sardi - e «strumentale» - in quanto può portare dei benefici simbolici e materiali (Banks 2012): il richiamo all'autenticità è strumentale alla legittimazione all'interno del campo del folklore e agli eventuali "profitti") - Tonio invece evoca (anche) l'autenticità come equivalente della verità, cioè di un'aderenza al testo coreutico archetipico che lui rinviene nel periodo a cavallo tra Ottocento e Novecento, ovvero il periodo grosso modo a cui arrivava la memoria dei testimoni più anziani da lui conosciuti tra gli anni '60 e '70 del secolo scorso' ${ }^{19}$. Tuttavia, Tonio non usa mai i termini filologia e filologico, tanto meno locuzioni come correttezza filologica e filologicamente corretto. Quando Tonio si vuole riferire al ballo che da ragazzino ha imparato dagli anziani, che poi ha approfondito e ricostruito attraverso le sue ricerche e che distingue da quello che propongono i gruppi folklorici, lui parla non di ballo "antico», ma di ballo «tradizionale», poiché parlare di un ballo antico significherebbe automaticamente riconoscere un ballo sardo «moderno», ossia avallare quello che fanno la maggior parte dei gruppi folklorici campidanesi:

Non c'è un ballo sardo moderno [ride; rido anche io]. Quando dicono: adesso facciamo il ballo sardo, il campidanese antico. No, non esiste il ballo campidanese antico. Esiste il ballo campidanese tradizionale. Moderno non ce n'è. Per forza deve essere antico, perché è una cosa vecchia, del passato, di tempo fa ${ }^{20}$.

Tonio nel 2000 organizza il suo primo corso di ballo sardo ${ }^{21}$. I corsi continuano ancora oggi e sono dedicati esclusivamente al ballo campidanese. Durano circa due ore e solitamente gli ultimi 20-30 minuti sono dedicati ad altri balli dell'Isola, più per divertimento e rilassamento che non per didattica. Infatti, è il momento della lezione in cui Tonio non deve più insegnare, poiché si tratta di balli molto noti che spesso gli allievi conoscono già, e in cui i partecipanti al corso, in un certo senso, si autogestiscono, visto che molte volte sono loro che scelgono i balli e, in qualche misura, autoapprendono.

Tutto un altro discorso è quello per il campidanese: la mezz'ora di balli cabesusesus o cabillus $^{22}$ per divertirsi la dice lunga, per contrasto, della complessità e della fatica associata all'apprendimento del ballo campidanese «tradizionale» ${ }^{23}$. Nel sistema coreutico sardo il ballo campidanese risulta il più complesso per il peculiare rapporto che si instaura tra la danza e la musica ${ }^{24}$. Secondo l'etnocoreologo Salvatorangelo Pisanu «Il caso del ballo campidanese è molto singolare dal punto di vista strutturale. Esso può raggiungere livelli di elaborazione molto alti [...]» (Pisanu 2013: 64). In questo ballo infatti è presente

[...] un rapporto stretto tra la parte musicale e quella coreutica che, per regola, non dovrebbe essere eseguita in altre parti musicali. Ed in effetti è questa la particolarità del ballo campidanese nella sua veste più sofisticata, il fatto che, anche se in modo non rigidamente previsto, vi è una preferenziale corrispondenza tra i tipi di Motivi musicali e i tipi di Motivi coreutici (Ibidem: 66).

Normalmente nelle danze sarde questo rapporto si articola nell'esecuzione di due tipi di passi: uno lento e posato (variamente denominato passu sèriu, a sa sèria, a passu ecc.) e uno veloce e vivace (passu alligru, passu lestru, a sa lestra ecc.), a cui corrispondono gli 
equivalenti passaggi musicali. Francesco Giannattasio e Bernard Lortat-Jacob li hanno distinti in «passo basso» e "passo alto» (Giannattasio, Lortat-Jacob 1982: 23-25). Quasi tutti i balli sardi, sono caratterizzati, per quanto riguarda il rapporto tra la parte coreutica e quella musicale, da una sorta di circolarità per la quale al passo basso segue il passo alto, dopo di che ritornerà il passo basso al quale seguirà di nuovo quello alto, $\mathrm{e}$ così via. Questa circolarità, peraltro, permette al ballerino e alla ballerina di prevedere l'andamento del ballo, a patto ovviamente che conoscano la danza e sappiano adattarsi alle eventuali personalizzazioni del suonatore ${ }^{25}$. Nel ballo campidanese, invece, il rapporto tra parti coreutiche e parti musicali è più ricco e diversificato, dal momento che sia i passaggi musicali che i passaggi coreutici sono più numerosi. Questo rapporto nel caso dei balli della Sardegna meridionale sembra che assuma una struttura lineare, cioè una successione progressiva di motivi musicali detta scala.

38 A questa progressione musicale corrisponde una progressione di motivi coreutici. Questi motivi sono più numerosi rispetto agli altri balli sardi e si dispongono come in una sorta di crescendo che culmina nei passi più complessi ed elaborati. Da questo punto di vista si può dire che, se la maggior parte dei balli sardi ha sempre una fase più vivace, a volte parossistica, che ritorna ciclicamente nel corso della stessa danza (la frequenza e l'intensità di queste fasi dipende dal suonatore che decide quanto far durare il ballo e dal rapporto di complicità e intesa che si può instaurare tra chi suona $\mathrm{e}$ chi balla), il ballo campidanese è forse il caso più emblematico tra quelle danze sarde in cui il culmine dell'esecuzione coreutica viene raggiunto intorno alla metà dell'esecuzione musicale o anche verso la fine, dopo che sono stati eseguiti tutti i passi necessari allo sviluppo della fase più elaborata e virtuosistica. In sintesi, secondo la ricostruzione fatta da Tonio Spiga, il ballo campidanese è strutturato in almeno sette passi diversi: su passu torrau (passo di tre battute in avanti e all'indietro); su passu 'e dusu (passo in due battute sulla stessa linea); su passu 'e tresi (passo in tre battute sulla stessa linea); su passixeddu (passo di una battuta in avanti); su passu apuntau (passo di una battuta sulla stessa linea da fermo); is fùrias (i passi più elaborati e dinamici, di pertinenza maschile); is sciampitas (il culmine virtuosistico delle furias e di tutto il ballo, anch'essi di pertinenza maschile) (cfr. Bentzon 2002: 73-76; Leoni 2012).

39 Alla conoscenza dei passi in rapporto alla musica va aggiunto il portamento appropriato (sètiu) e la capacità di eseguire un leggero movimento sussultorio (sàntziu). Questa complessità dipende anche dal rapporto, per così dire, elettivo che c'è tra il ballo campidanese e le launeddas, lo strumento musicale più importante tra quelli che creano la musica per il ballo ${ }^{26}$. Tra gli strumenti sardi di tradizione orale, le launeddas presentano il repertorio più ricco e complesso per quanto riguarda le musiche da ballo, complessità che si riflette, appunto, nella stessa esecuzione della danza. Infatti un'ulteriore articolazione dei balli campidanesi consiste nel fatto che la scala non è unica, ma cambia a seconda dell'accordatura delle launeddas. Poiché esistono una decina di accordature diverse (cuntzertus), ed ogni accordatura distingue un tipo di launeddas (mediana a pipia; fiuda bagadia; puntu 'e organu; fioràssiu ecc.), esistono scale differenti che determinano balli differenti. Per cui abbiamo tanti balli campidanesi quanti sono i cuntzertus, i quali danno anche il nome ad ogni singola danza: ballu a mediana; ballu a fiuda bagadia; ballu a puntu' 'e organu; ballu a fioràssiu ecc.).

40 I danzatori e le danzatrici devono dunque, in linea di massima, saper riconoscere la scala di ogni cuntzertu per eseguire al meglio i balli. «La musica te lo dice» ${ }^{27}$ : così Tonio sintetizza questo rapporto tra la danza e la musica. Devi capire la musica per sapere che 
passi devi fare. Tuttavia, non bisogna dimenticare quell'aspetto del ballo che è dato dall'originale apporto personale di ogni danzatore e di ogni suonatore. Ogni sonada, ovvero la singola esecuzione musicale, si distingue per l'interpretazione che il suonatore da della scala. I danzatori, da parte loro, se sanno capire quello che la musica gli dice, possono a loro volta interpretare in maniera personale e assai varia la sonada. Il ballo campidanese allora sembrerebbe che proprio per via della sua estrema elaborazione coreutica e musicale permetta una libertà di espressione personale sorprendente ${ }^{28}$ :

Perché poi puoi fare tante variazioni tu, puoi fare tante varianti, nell'ambito del ballo te lo crei anche tu. $\mathrm{E}$ anche il suonatore può seguire la scala vera e propria oppure può anche non seguire una scala perfetta, però può seguirla un po' a suo modo, perché si può fare anche così volendo, però l'importante è che sia una suonata ordinata, che sia ordinata, che faccia ballare bene. Quindi sono tante, tante possibilità. Quasi quasi non finiscono mai le possibilità del [parole non capite]. [...]. Perché è un tipo di musica composta in un modo che tu puoi fare quello che vuoi, però sempre tornando a passo con la musica, puoi fare quasi tutto quello che vuoi. $\mathrm{E}$ ogni ballerino si distingueva l'uno dall'altro ballando, perché ognuno poi gli dà la sua impronta personale ${ }^{29}$.

Questa dialettica tra la necessità strutturale che la musica impone alla danza e la libertà delle pratiche concrete dei danzatori da luogo a uno spazio coreutico del quale è bene comprendere dove termina il vincolo delle strutture oggettive e dove iniziano le possibilità della performance soggettiva di coloro senza i quali la danza non esisterebbe, ovvero le danzatrici e i danzatori: «sempre tornando a passo con la musica», come dice Tonio, potrebbe essere la formula che esprime la fedeltà ultima alla musica, ma dopo che magari, nei limiti consentiti, è stata "tradita" con altri passi (cfr. Pisanu 2012: 91) ${ }^{30}$.

\section{Dall'egemonia alla resistenza}

La posizione di Tonio nella scena locale è eccentrica: emergente e marginale allo stesso tempo. Come ho detto, le sue idee e il suo operato si collocano su fronte dei filologi del ballo campidanese: egli è di fatto il capofila di questa linea, essendo il massimo maestro riconosciuto in materia di ballo campidanese. Tuttavia la sua è una guida e una preminenza implicita. Innanzitutto si colloca in una posizione di minoranza: se da una parte ha la stima, l'ammirazione e il riconoscimento da parte dei cultori del ballo campidanese «tradizionale», dall'altra la sua proposta è di fatto confinata ai margini della scena dalla grande maggioranza dei gruppi folklorici locali che hanno scelto altri approcci. Pochissimi gruppi hanno accolto la sua proposta della danza e il suo insegnamento. Quasi tutti i gruppi della zona hanno optato per esecuzioni più semplici e allo stesso tempo più spettacolari: il ballo campidanese standard attuale, così come è proposto dai gruppi folklorici, è il risultato di una stilizzazione del passo base, dell'introduzione di nuove coreografie e di un diverso rapporto con la musica, anche perché questa spesso non è più eseguita con le launeddas o con la fisarmonica, ma con l'organetto.

Un ulteriore corollario è che Tonio non fa parte di nessun gruppo folklorico, tanto meno ne ha creato uno secondo i suoi criteri, a parte un tentativo di breve durata negli anni ' 80 , e quindi non avendo alle spalle nessuna associazione organizzata e preferendo una via minoritaria al ballo sardo ha uno scarso, per non dire nullo, appoggio 
istituzionale e poche possibilità di attrarre risorse. La sua proposta filologica si esplica fondamentalmente attraverso i corsi di ballo, a volte appoggiandosi a qualche gruppo folklorico amico che gli mette a disposizione la sede, altre volte usufruendo di spazi messi a disposizione dalle amministrazioni locali per iniziative culturali o ricreative. I suoi corsi non rientrano comunque nell'ambito di una associazione culturale formalmente riconosciuta che possa costituire un punto di riferimento stabile come lo sono i gruppi folklorici. Anche se i suoi corsi hanno luogo quasi ogni anno con una certa regolarità. Saltuariamente frequenta qualche gruppo locale per via di amicizie o perché gli viene chiesta una consulenza: raramente questi rapporti sono sfociati nella completa adozione del "suo" ballo. Inoltre capita a volte che Tonio con i suoi allievi più assidui venga invitato ad esibirsi in occasione di festival, feste o eventi pubblici. Attualmente sta insegnando il ballo campidanese ad alcuni gruppi folklorici locali: viste le ambivalenti esperienze del passato e la necessità di un lungo apprendistato, è ancora presto per dire se poi il ballo verrà inserito stabilmente nel repertorio di questi gruppi ed eseguito negli spettacoli. Anche se qualche gruppo sta già iniziando a portare sul palco i primi frutti di questo lavoro. Infine c'è la personalità di Tonio che è tutt'altro che predisposta ad ambizioni da leader e a giocare ruoli di primo piano sulla scena pubblica: direi che Tonio unisce mitezza di carattere e intransigenza coreutica.

Dunque si può dire che Tonio possiede un basso capitale politico ed economico, da una parte, e un elevato capitale culturale, dall'altra. Devo tuttavia precisare che cosa intendo in questo caso per capitale culturale, dato che i titoli di studio di Tonio non vanno oltre il diploma di scuola superiore (per la precisione, il diploma di maturità artistica). Infatti preferisco ribattezzarlo capitale culturale subalterno. Ovvero quell'insieme di conoscenze e di competenze acquisite, riconosciute e impiegate in contesti che non sono contemplati dall'educazione e dall'istruzione ufficiali. Questi contesti riguardano saperi non identificabili con la cultura egemone, ma nemmeno con una generica costellazione culturale tradizionale immaginata come nettamente opposta alla prima, e quindi, in qualche misura, reificata: si tratta semmai di un segmento culturale specifico in quanto parte di un orizzonte simbolico e materiale più ampio $\mathrm{e}$ dinamico quale può essere quello ascrivibile alle culture popolari e proprie dei subalterni ${ }^{31}$. Così, un ballerino di danza classica possiede un capitale culturale relativo alla danza classica, dal momento che questa è insegnata nelle accademie di danza ufficialmente istituite e riconosciute, e questo capitale è spendibile sia nel campo specifico della danza che in qualunque curriculum professionale culturale. Mentre un danzatore di ballo sardo non possiede un equivalente culturale ufficialmente riconosciuto (non esistono abilitazioni all'insegnamento del ballo sardo), ma il suo sapere pratico di ballerino può essere fatto valere, perché riconosciuto e riconoscibile, nel sotto-campo dalla danza sarda in particolare e nel campo del folklore in generale ${ }^{32}$. Pertanto Tonio è in possesso di un elevato capitale culturale subalterno, ovvero un'alta competenza esecutoria del ballo sardo e una vasta conoscenza empirica del mondo del ballo campidanese. Discutendo della sua posizione all'interno della sotto-scena del ballo, egli sottolinea l'aspetto conoscitivo del suo particolare capitale culturale, nonché della sua doppia valenza pratica e teorica:

Adesso nell'ambito della conoscenza sono pochi quelli che sanno quello che so io. Quindi in effetti... Però non so, magari vivendo nel periodo in cui si ballava veramente ce ne potevano essere anche più bravi di me, sicuramente, capito? Io faccio quel poco che so fare, quello che conosco cerco di farlo bene, ma certe cose sono sicuro che sono così. Poi dipende dalla mia passione se riesco a farlo bene, se piace o non piace. Ma mi dicono che lo faccio bene ${ }^{33}$. 

correttezza del ballo che eseguono in pubblico. Tuttavia, almeno da parte sua, il fatto di occupare posizioni differenti e di avere delle prese di posizione divergenti non si tramuta in conflitti espliciti. Tonio ha preferito una contrapposizione implicita, leggibile nelle cose che fa piuttosto che nelle cose che dice o nel modo in cui le dice. Il suo è un atteggiamento quasi stoico: «A me non interessa proprio niente delle rivalità» ${ }^{35}$. $\mathrm{E}$ sembra che riposi sulla convinzione di aver cercato la verità e di aver agito correttamente: «Il mio rapporto con i gruppi folk è un rapporto amichevole, ma gli ho lasciato fare un po' a loro, chi ha voluto ascoltare ha voluto ascoltare, chi non ha voluto ascoltare non ha voluto ascoltare. Lo sanno com'è, solo che non hanno la voglia di farlo, tutto là» ${ }^{36}$. Così il rapporto di Tonio con i gruppi folklorici locali si regola sulla base di parametri estetici extra-filologici, non scevri a volte da implicazioni di natura amicale, e allo stesso tempo sul principio della tradizionalità come baricentro ideologico della sua pratica filologica:

Ci sono gruppi così sofisticati che lo fanno bene, però sofisticati, nella loro sofisticazione; siete bravi inventandovi le cose, guardandovi lo fatte bene, quello che fatte lo fatte bene, ordinato, però non siete tradizionali, non è ballo sardo: glielo detto, sopratutto al gruppo di [...]. Sì li posso apprezzare, posso anche apprezzarli per quello che fanno, posso apprezzare anche [...], per le scenografie che fanno, possono anche piacermi a livello così, però se poi devo giudicarli dal lato culturale $\mathrm{e}$ tradizionale, no, cambia tutto ${ }^{37}$.

Possiamo quindi dire che questa relazione va da una stoica indifferenza a una cortese ostilità, passando in alcuni casi per dei rapporti di amicizia dove ognuno rispetta le differenti scelte dell'altro. In generale comunque il rapporto è quello di una contrapposizione di linee di pensiero e di pratiche coreutiche, la cui conflittualità è oggettivamente, ma implicitamente, inscritta nelle relazioni che disegnano la mappa del campo di forze della scena del ballo sardo in area basso-campidanese. Ciò che sostengo è che Tonio, con i suoi allievi, rappresenta una sorta di cellula dissidente all'interno della scena locale e lo fa dando una curvatura resistente al filologicamente corretto in un contesto dove il richiamo costante all'autenticità della tradizione sembra al servizio dello status quo e di posizioni consolidate, ossia di una sorta di egemonia locale che ha strutturato il senso comune nella scena delle danze folkloriche nella Sardegna meridionale.

In effetti anche Tonio, come tutti gli attori della scena, è mosso dall'illusio dell'esistenza e del valore indiscussi della tradizione, ma la sua soggettività e la sua agentività in un certo senso spiccano, o si distinguono, perché improntate a una declinazione della legge fondamentale del campo in una direzione che è controcorrente: si direbbe che nessun altro come lui si è adoperato pubblicamente per un ballo campidanese che fosse come quello che facevano is antigus (gli antenati). Mentre invece la tendenza 
maggioritaria, almeno nella Sardegna meridionale, è la rappresentazione di un ballo molto stilizzato e spettacolare: esattamente quell'egemonia locale che Tonio contesta. Tuttavia, Tonio rimane ben dentro il campo poiché è partecipe della stessa illusio che muove tutti gli attori della scena, tradizionalisti e modernisti, cioè la credenza nell'esistenza di una tradizione e della necessità di preservarla: in un certo senso lui spinge questa convinzione agli estremi per quanto riguarda il ballo sardo. La stessa filologia è un altro meccanismo messo in funzione dal campo del folklore in quanto dispositivo patrimoniale e in quanto cultura riflessiva: esso mette per la prima volta a disposizione di svariati soggetti una strumentazione retorica, discorsiva e conoscitiva quali possono essere forme di sapere come la storiografia, l'etnografia e appunto la filologia. Gli attori della scena mettono al servizio dell'illusio questi strumenti (e ovviamente così facendo anche al servizio di propri specifici obiettivi e interessi). Ma Tonio forse prende più sul serio l'illusio, o più alla lettera. Questo fa appunto di lui una figura eccentrica nella scena del folklore campidanese e, in un certo senso, un perturbatore delle politiche locali del patrimonio culturale. Il suo approccio filologicocritico si distingue, neanche a dirlo, da quello scientifico dell'antropologia, della storiografia e della filologia accademiche, ma anche da quello proclamatore e retorico delle istituzioni, con le quali i suoi rapporti si direbbero ridotti al minimo.

In effetti, il paradigma del filologicamente corretto si presenta come un prisma sfaccettato da diversi tagli tattico-strategici. Se Palumbo ne ha innanzitutto messo in luce il lato colto e istituzionale insieme alle retoriche rivendicative locali e gli ha fatto compiere uno spostamento da risorsa conoscitiva a oggetto stesso della conoscenza antropologica, io dal mio canto propongo di considerarne una ulteriore declinazione, cioè quella che vede una pratica filologica intrecciata a una volontà di verità (qual è il vero ballo sardo campidanese?, si chiede in fondo Tonio) che in un determinato contesto e in determinate condizioni (quello creato dal campo del folklore organizzato e dagli strumenti che mette a disposizione) risulta ribelle e perturbante rispetto allo stato di cose presenti a livello locale (una scena dominata da un ballo sardo stilizzato e dai gruppi folklorici che possono contare del supporto logistico ed economico delle istituzioni).

Si tratta dunque di tattiche - intese come unico spazio di manovra che le condizioni del terreno in cui ci si muove, degli strumenti a disposizione e dei rapporti di forza consentono (De Certeau 2001: 69-75) - che possiamo vedere all'opera dentro e ai margini del campo del folklore e del sotto-campo del ballo. Tale campo istituisce limiti e confini discorsivi, limiti e confini pratici, ma proprio per questo crea anche le condizioni per nuove condotte o per condotte anomale. Perciò ritengo che un maestro di ballo sardo come Tonio Spiga imprime al filologicamente corretto una traiettoria che non è più quella disegnata dal sapere colto e dalle retoriche istituzionali proprie del paradigma patrimonialista. E nemmeno quella di chi, a livello locale, sfrutta questa categoria per legittimare il proprio operato agli occhi delle agenzie politiche e culturali ufficiali. Bensì è il filologicamente corretto che stavolta sta al fianco di soggetti minoritari che intrattengono una lotta per la verità in un campo dove la verità più che la posta in gioco sembra essere lo strumento per raggiungere determinati obiettivi. È quindi un filologicamente corretto che transita dal piano dell'egemone a quello del subalterno e che su questo terreno assume una posizione di micro-resitenza nei confronti di una micro-egemonia locale. Resta da vedere come si concluderà questa 
lotta. Anche se, pur essendo al momento impari, c'è da credere che durerà finché ci sarà un ballo che chiameremo ballu sardu.

\section{Conclusioni}

51 Il caso che qui ho riportato descrive una traiettoria del filologicamente corretto così come viene interpretato e incarnato da determinati soggetti (cultori tradizionalisti del ballo sardo campidanese), in un determinato contesto (il sotto-campo del ballo sardo all'interno del campo del folklore nella Sardegna meridionale). Mi è sembrato importante mettere in evidenza la particolare declinazione di una categoria che aveva già avuto il merito, nell'uso proposto da Palumbo, di svelare i sottintesi ideologici indiscussi nei discorsi e nell'operato di certi settori della politica patrimonialista. Ritengo che adesso questa categoria possa essere considerata anche per il ruolo che gioca all'interno dello stesso campo della cultura popolare contemporanea e soprattutto in quanto principio su cui far leva per opporsi alle rappresentazioni dominanti della storia e dell'identità locali. Ma se la forma che credo di aver rilevato del filologicamente corretto può avere una qualche importanza, penso ce l'abbia nella misura in cui ha qualcosa da dire in merito a temi più complessivi, tra i quali quelli della riflessività, della transculturazione e della polemologia nell'ambito della cultura popolare odierna.

Se ho provato a seguire le traiettorie del filologicamente corretto non è solo perché mi è parso che esse potevano mostrarci delle forme di indigenizzazione, ma anche perché queste traiettorie ci conducono probabilmente in territori dove è possibile vedere in atto processi di transculturazione: la filologia popolare di Tonio Spiga può forse essere considerata un'appropriazione subalterna di saperi egemoni riadattati all'orizzonte sociale di riferimento in cui egli si muove. In fondo Tonio e i suoi allievi ripropongono dall'interno della scena del folklore quell'approccio filologico a cui prima venivano, e ancora oggi vengono, sottoposti danzatori come loro: essi si collocano in quella particolare posizione per la quale sono, da una parte, depositari e continuatori locali di una tradizione subalterna (sebbene nella forma cosciente di ciò che un tempo costituiva un'esperienza vissuta ma non tematizzata) e, dall'altra, utilizzatori di categorie e strumenti globali di provenienza egemone (seppure entro i limiti delle proprie cornici culturali di riferimento).

Il filologicamente corretto compie così una serie di traiettorie che lo portano dalla storia dell'arte e dalla letteratura all'antropologia e al folklore per poi arrivare ad appropriarsene gli stessi soggetti che della filologia erano l'oggetto. La riflessività culturale diventa allora un tratto della nuova cultura popolare che è da tempo impegnata a dare un significato al passato per trasmetterlo al presente (cfr. Bauman 1992: 32). Questo sforzo semantico si lega a quella «giustificazione ad esistere» che gli individui ritrovano in ultima istanza nell'indiscusso interesse al gioco che giocano nel campo o in più campi che li impegnano nella loro vita (Bourdieu 1998: 248-251). Il campo del folklore peraltro costituisce uno spazio tendenzialmente dedicato al piacere e alla gioia, dal momento che, almeno in astratto, sono queste le finalità della danza $\mathrm{e}$ della musica, e in particolare della festa quale luogo elettivo di queste espressioni culturali. Esso è uno di quei luoghi e di quei momenti in cui le persone delle classi popolari danno un significato piacevole alla loro vita, e questo piacere è altamente relazionale, cioè orientato verso l'altro (Walker, Kavedzija 2015). 
Tuttavia, l'idillio folklorico è sovente rotto dalle turbolenze e dal conflitto. Ciò costituisce probabilmente una sorta di scandalo ontologico: laddove si celebra il piacere di vivere si riproduce la discordia e la lotta. Il campo del folklore infatti sembra mantenuto, oltre che dall'illusio comune, da una serie di forze che opponendosi creano le condizioni per la costituzione di quelle relazioni di posizioni oggettive che in definitiva formano il campo: il conflitto e la divergenza, non meno dell'armonia e del consenso, sembrano mantenere vivo e vivace il campo del folklore sardo. L'alto contenuto relazionale del piacere e della condivisione si intreccia allora con l'altrettanto alto contenuto relazionale della discordia e dello scontro. La traiettoria assunta dal filologicamente corretto nel sotto-campo del ballo sardo in area bassocampidanese ci racconta anche di questo: di una postura oggettivamente resistente rispetto allo status quo coreutico e di un conseguente conflitto a bassa intensità. Se dunque le traiettorie del filologicamente corretto conducono anche al conflitto e alla resistenza, forse significa che bisogna continuare ad esplorare il tema di una polemologia del culturale e del popolare, di quello scandalo per il quale sembra che, nella vita sociale, ordinaria e straordinaria, la pace e la guerra si inseguano a vicenda.

\section{BIBLIOGRAFIA}

Agamben G. 2010 Categorie italiane. Studi di poetica e di letteratura, Laterza, Bari-Roma.

Appadurai A. 2001 Modernità in polvere. Dimensioni culturali della globalizzazione, Meltemi, Roma [ed. or. 1996].

Banks M., 2012 «True to Life: Authenticity and the Photographic Image», in Fillitz, T., Saris J. (eds) Debating Authenticity: Concepts of Modernity in Anthropological Perspective, Berghahn Books, Oxford: 160-171.

Bauman R., 1992 «Folklore» in Id. (ed.), Folklore, Cultural Performances, and Popular Entertainments. A Communications-centered Handbook, Oxford University Press, New York, Oxford: 29-40.

Bauman R., 2008 «The Philology of the Vernacular», in Journal of Folklore Research, vol. 45, n. 1: 29-36.

Bendix R., 1988 «Folklorism: The challenge of a concept», in International Folklore Review, 6: 5-15.

Bendix R., 1997 In Search of Authenticity: The Formation of Folklore Studies. University of Wisconsin Press, Madison.

Bentzon A.F.W., 2002 Launeddas, Iscandula, Cagliari [ed. or. 1969].

Bortolotto C., 2007 «From Objects to Processes: UNESCO's Intangible Cultural Heritage», in Journal of Museum Ethnography, n. 19: 21-33.

Bortolotto C., 2013 «Authenticity: A Non-Criterion for Inscription on the Lists of UNESCO's Intangible Cultural Heritage Convention», in Final Report of 2013 IRCI Meeting on ICH - Evaluating the Inscription Criteria for the Two Lists of UNESCO's Intangible Cultural Heritage Convention, 
International Research Centre for Intangible Cultural Heritage in the Asia-Pacific Region (IRCI), Osaka: 73-79.

Bourdieu P., 1995 Ragioni pratiche, il Mulino, Bologna.

Bourdieu P., 1998 Meditazioni pascaliane, Feltrinelli, Milano.

Bourdieu P., Wacquant L. J. D., 1992 Risposte. Per un'antropologia riflessiva, Bollati Boringhieri, Torino.

Calzia F., 2014 «Ballos sardos in pratza. La nuova stagione delle danze nell'isola», in Analitica. Rivista on line di studi musicali, n.7: 89-101.

Cirese A.M., 1973 Cultura egemonica e culture subalterne. Rassegna di studi sul mondo popolare tradizionale, Palumbo, Palermo.

Contini G., 1986 Breviario di ecdotica, Einaudi, Torino.

De Certeau M., 2001 L'invenzione del quotidiano, Edizioni Lavoro, Roma [ed. or. 1980].

Deiana A., 2012 «Né innocente, né innocuo: il folklore tra volontà di sapere, politiche del quotidiano e storia del presente. Ovvero le istruttive vicissitudini del rapporto tra antropologi e gruppi folklorici», in Archivio di Etnografia, n.s., a. VII, vol. I: 75-94.

Deiana A., 2013 «Il culturale in Sardegna. Un altro sguardo da un s/oggetto inedito: i gruppi folkloristici», in Studi e ricerche, vol. VI: 141-153.

Deiana A., 2017 Effetto folklore. Usi e significati della tradizione nella Sardegna contemporanea, Aracne, Roma.

de Sanctis Ricciardone P., 2007 Ultracorpi. Figure di cultura materiale e antropologia, Liguori, Napoli.

Dutton D. , 2003 «Authenticity in Art», in Levinson J. (ed.), The Oxford Handbook of Aesthetics, Oxford University Press, New York: 258-274.

Faubion J., 1993 Modern Greek Lessons. A Primer in Historical Constructivism, Princeton University Press, Princeton.

Gala G., 2000 «Il ballo sardo tra folklore e folklorismo. Tipologia dei balli etnici e trasformazione spettacolare in Sardegna», in AA.VV., Il ballo sardo: storia, identità e tradizione. II: Forme e contesti del ballo sardo, Taranta, Firenze: 19-75.

Gala G., 2004 A passu. Appunti di antropologia del ballo sardo, Taranta, Firenze.

Gallini C., 1971 Il consumo del sacro. Feste lunghe di Sardegna, Laterza, Roma-Bari.

Giannattasio F., Lortat-Jacob B., 1982 «Modalità di improvvisazione nella musica sarda: due modelli», in Culture musicali, I: 3-35.

Gramsci A., 1975 «Osservazioni sul «Folclore»», in Id., Quaderni del carcere, Einaudi, Torino [1929-1935]: 2311-2317.

Handler R., 1988 Nationalism and the Politics of Culture in Quebec, University of Wisconsin Press, Madison.

Hannerz U., 2001 La diversità culturale, il Mulino, Bologna.

Kilani M., 1992 La construction de la mémoire. Le lignage et la sainteté dans l'oasis d'El Ksar, Labor et Fides, Genève. 
Leoni A., 2012 «Sonadoris e baddadoris, sonadas e passus», in Casu F., Lutzu M. (a cura di) Enciclopedia della musica sarda, vol. 11: Launeddas, a cura di M. Lutzu, L'Unione Sarda, Cagliari: 62-69.

Lindholm, Ch., 2013 «The Rise of Expressive Authenticity», in Anthropological Quarterly, vol. 86, n. 2: 361-396.

Linnekin, J. , 1983 «Defining Tradition: Variations on the Hawaiian Identity», in American Ethnologist, vol. 10, n. 2: 241-252.

Lutzu M. (a cura di), 2012 Launeddas, voll. 11-12, in Casu F., Lutzu M. (a cura di), Enciclopedia della musica sarda, L'Unione Sarda, Cagliari.

Marras M., 2003 Un paese in ballo. Danza e società nel Carnevale seneghese, Condaghes, Cagliari.

Mirizzi F., 2007 «Gruppi folkloristici e valorizzazione delle culture locali», in Parbuono D. (a cura di), Folcloristico senza timore. I cinquant'anni dell'“Agilla e Trasimeno" e l'Italia del folklore, Castiglione del Lago, Edizioni Duca della Corgna: 59-65.

Nahachewsky A., 1995 «Partecipatory and Presentational Dance as Ethnochoreological Categories», in Dance Research Journal, 27/1: 1-15.

Orrù L., 1999 Maschere e doni, musiche e balli. Carnevale in Sardegna, Cuec, Cagliari.

Palombini C., 2007 «Insegnare il folklore? Appunti metodologici per i gruppi folcloristici», in Parbuono D. (a cura di), Folcloristico senza timore. I cinquant'anni dell'“Agilla e Trasimeno" e l'Italia del folklore, Castiglione del Lago, Edizioni Duca della Corgna: 49-53.

Palumbo B., 2003 L'Unesco e il campanile. Antropologia, politica e beni culturali in Sicilia orientale, Meltemi, Roma.

Parodi M., 2011 Il campo del folklore tra valorizzazione e integrazione culturale. Il caso di São Luis del Maranhão, Tesi di Dottorato, Università degli studi di Milano Bicocca.

Pisanu S., 2000 «Un'ipotesi di analisi del ballo sardo attraverso una lettura etnomusicologica», in AA.VV., Il ballo sardo: storia, identità e tradizione. II: Forme e contesti del ballo sardo, Taranta, Firenze: 151-174.

Pisanu S., 2012 «Il ballo a launeddas», in Casu F., Lutzu M. (a cura di), Enciclopedia della musica sarda, vol. 12: Launeddas (a cura di M. Lutzu), L'Unione Sarda, Cagliari: 88-93.

Pisanu S., 2013 Ballare in Sardo. Anatomia e geografia di un linguaggio coreutico, Edes, Sassari.

Pratt M. L., 1992 Imperial Eyes. Travel Writing and Transuculturation, Routledge, London and New York.

Thornton S., 1998 Dai club ai rave. Musica, media e capitale sottoculturale, Feltrinelli, Milano.

Walker H., Kavedzija I., 2015 «Values of Happiness», in Hau: Journal of Ethnographic Theory, 5(3): 1-23.

\section{NOTE}

1. «La lettura più difficile è la più forte»: principio filologico della critica testuale.

2. Parte della ricerca etnografica su cui si basa questo scritto è stata fatta nell'ambito del progetto "Beni demoetnoantropologici: saperi, memorie, musei come risorse per il presente" (finanziato dalla Regione Autonoma della Sardegna, legge 7/2007), coordinato in qualità di responsabile scientifica da Gabriella Da Re e svoltosi tra il 2013 e il 2014. Ringrazio Marco Lutzu e 
Angelo Pisanu per i primi preziosi riscontri sul testo in fase di stesura. Così come ringrazio i due revisori anonimi i cui commenti sono stati molto utili per il miglioramento dell'articolo.

3. Per evitare determinazioni essenzialiste come "tradizionale" e "popolare", userò il termine emico ballo sardo per intendere l'intero sistema coreutico sardo.

4. A questo riguardo non posso che rimandare a studi prettamente etnocoreologici (Gala $2000 \mathrm{e}$ 2004; Pisanu 2000 e 2013). Tuttavia, è bene che il lettore sappia che quando parliamo di balli sardi si tratta, da un punto di vista formale, di un sistema coreutico che si distingue per la sua «struttura modulare», ovvero per la «stretta corrispondenza fra metrica coreutica e metrica musicale [...]»; l'«impianto coreografico basilare circolare: con incatenamento dei balladores in cerchio chiuso e introverso» [...]; la postura «a busto eretto ma non rigido e scarsa mobilità della parte superiore [...] altamente compensata da un'estrema vivacità sino ad un virtuosismo dinamico di gambe e piedi»; la «connessione obbligatoria con stretto legame degli arti superiori (mani e/o braccia) tra i ballerini»; la «[...] frammentazione delle cadenze ritmiche e conseguente tremolio sussultorio» (Gala 2004: 17-18).

5. Per una considerazione di questa tematica in riferimento a uno studio di caso si veda Marras 2003.

6. La novità costituita dalle scuole di ballo sardo meriterebbe una trattazione a parte. Qui mi limito a precisare che non si tratta di scuole di danza stabili e registrate come esercizi commerciali, ma di corsi estemporanei che ruotano intorno alle figure di ballerini/e insegnanti che si premurano di prendere in affitto un locale pubblico o privato per il tempo dei corsi. In alcuni casi chi allestisce questi corsi ha deciso di formare un'associazione, cosicché i partecipanti risultano iscritti all'associazione, nonché assicurati. Normalmente gli iscritti alle scuole pagano una quota mensile che serve a coprire le spese di affitto dei locali. Dipende da come sono pensati e organizzati i singoli corsi, che solo nell'area di Cagliari sono una decina, se queste quote comprendono o meno una percentuale di ricavo per l'insegnante.

7. Di fronte a quella che sembra la rarità e la peculiarità del concetto di cui stiamo parlando, avanzo l'ipotesi che il filologicamente corretto sia una categoria italiana, per prendere in prestito una formula con la quale Giorgio Agamben ha indicato le polarità costitutive della cultura letteraria italiana (le «categorie italiane», appunto) (Agamben 2010), ossia una categoria che scaturisce dalla particolare strutturazione del contesto culturale italiano, in particolare quella relativa al patrimonio storico-artistico.

8. Non essendomi mai imbattuto in questo particolare uso del concetto di filologicamente corretto nella letteratura antropologica da me frequentata, a parte il lavoro di Palumbo, non potevo fare altro che verificare le occorrenze di questa locuzione in archivi elettronici come Jstor. Ma neanche in un archivio del genere risultano studi che trattino questa declinazione del concetto. A parte il suddetto articolo di Bortolotto.

9. Per una genealogia del campo del folklore in Italia rimando a Deiana 2017. Per un più marcato uso etnografico della categoria bourdieusiana di campo in riferimento al folklore si veda Parodi 2011.

10. In realtà $\mathrm{i}$ soggetti in campo sono di più. A partire dai suonatori, passando per gli organizzatori e curatori degli spettacoli e delle serate di ballo, fino alle televisioni che mandano in onda trasmissioni espressamente dedicate ai balli sardi in costume. Ma qui ho voluto privilegiare quei soggetti che incarnano letteralmente la danza, in fondo secondo l'idea espressa in un verso di William B. Yeats: «Come possiamo distinguere il danzatore dalla danza?». Mentre invece possiamo distinguere il danzatore e la danza dal suonatore, da un comitato festivo, o dal presentatore di uno spettacolo folklorico.

11. Si pensi ai rapporti tra i gruppi folklorici e le amministrazioni locali, le pro loco, i comitati festivi e le istituzioni regionali.

12. Da tempo ormai i gruppi folkloristici parlano di una «crisi del folklore» per dire che non ci sono più a disposizione le risorse di una volta, grosso modo l'epoca che va dagli anni '60 agli anni 
'90 del secolo scorso, quando l'ESIT (Ente Sardo Industrie Turistiche) finanziava i gruppi folklorici per promuovere l'immagine della Sardegna. Va detto comunque che queste risorse contribuivano a potenziare l'attività dei gruppi folklorici e il prestigio dei loro dirigenti più che ad arricchire singole persone nella scena del folklore. Anche se non mancano le voci di chi sostiene che qualcuno nella scena ne ha ricavato profitti economici non indifferenti.

13. Uso la parola 'maestro' nella doppia accezione di colui che ha acquisito una maestria in un dato ambito del saper-fare e che poi insegna questo saper-fare ad altri. Paradossalmente l'appellativo di maestro della danza è più calzante oggi in un'epoca di rarefazione della pratica $\mathrm{e}$ delle competenze piuttosto che per i tempi in cui il ballo sardo costituiva un insieme di pratiche e di competenze diffuse: ieri non c'era bisogno di maestri, oggi invece sì.

14. Il ballo sardo campidanese viene chiamato così per via della zona in cui viene praticato. Grosso modo si tratta di un'area che comprende tutta la pianura del Campidano da Cagliari fino a Oristano, ma quasi come per la variante linguistica del sardo, cioè il campidanese, questa area coreutica si estende nel sud-ovest della Sardegna (nelle regioni del Sulcis e dell'Iglesiente) e nel sud-est (nelle regioni della Trexenta, del Parteolla e del Sarrabus). Tuttavia, questa macro area non è uniforme da un punto di vista coreutico e in realtà è meglio parlare di balli campidanesi. Il ballo del Basso Campidano è uno di questi balli. Geograficamente il Basso Campidano è quello intorno a Cagliari e i paesi vicini, ma da un punto di vista coreutico ancora una volta l'area va allargata fino a lambire il medio Campidano e il Sulcis e a comprendere il Parteolla, la Trexenta e il Sarrabus (Cfr. Bentzon 2002: 74). Anche in questo caso non possiamo parlare di perfetta omogeneità del ballo, ma le variazioni locali non sono tali da costituire differenti tipologie coreutiche: le caratteristiche fondamentali rimangono invariate (rapporto tra motivi musicali e motivi coreutici, il tipo di presa con le mani tra ballerini, la musica eseguita in particolar modo con le launeddas, ma anche con altri strumenti quali flauti di canna, chitarra e fisarmonica che ne riprendono le melodie e i principi strutturali).

15. Intervista del 19/12/2013.

16. Intervista del 19/12/2013.

17. Intervista del 19/12/2013.

18. Intervista del 19/12/2013.

19. Intervista del 19/12/2013.

20. Intervista del 19/12/2013.

21. È in quell'occasione che l'ho conosciuto. A partire da quell'anno terrà i suoi corsi a cadenza quasi annuale. Io ho partecipato più o meno regolarmente ai corsi fino al 2004. Ma la frequentazione con Tonio è continuata per motivi di ricerca, per una comune passione per il ballo sardo e, ormai, anche per amicizia.

22. Questi due termini nella Sardegna meridionale identificano tutto ciò che appartiene alla Sardegna centrale e settentrionale, comprese le danze. A seconda del contesto di enunciazione possono avere delle sfumature positive, negative o neutre (non di rado scherzose).

23. Come vedremo subito, l'aspetto più complicato per chi vuole imparare i balli campidanesi è la comprensione del complesso rapporto tra motivi musicali e motivi coreutici. Mauro Frigau, allievo di Tonio e assai impegnato nello studio del ballo campidanese, sia per quanto riguarda l'esecuzione coreutica che l'acquisizione della conoscenza della musica, mi dice che, quando si tratta di identificare i passi corretti con i passaggi musicali di più difficile interpretazione, è uno studio che comporta «una grande sofferenza» (conversazione estemporanea durante il corso di ballo, 02/12/2016).

24. Le descrizioni del ballo sardo campidanese che seguiranno non hanno alcuna pretesa di esaurire la vastità dell'argomento e tanto meno di affrontarne tutte le implicazioni etnocoreologiche. Questa brevissima introduzione al ballo campidanese servirà esclusivamente a mostrare le peculiarità di questa danza in rapporto all'approccio filologicamente corretto così come è espresso da Tonio Spiga e quindi in relazione al tema della mia ricerca. Per un 
approfondimento rimando a Leoni 2012 e Pisanu 2012. Inoltre non è certo mia intenzione instaurare delle gerarchie tra i balli sardi, ognuno portatore della sua propria specificità, complessità e bellezza, fermo restando l'unanime riconoscimento, tra gli studiosi e i ballerini e le ballerine più esperte, del caso particolare rappresentato dai balli campidanesi all'interno del sistema coreutico sardo.

25. Va precisato comunque che questo modello è tutt'altro che rigido e uniforme. A partire dall'esistenza in molti balli di passi intermedi che fungono quasi da preparazione o anticipazione del passo più vivace o elaborato. Inoltre va tenuto conto della variabile rappresentata dai singoli danzatori e suonatori: i primi non sempre sono tenuti a eseguire i passi più elaborati o saltellati, mentre i secondi possono sia apportare delle interpretazioni che fare delle improvvisazioni impreviste per i ballerini e le ballerine.

26. Le launeddas sono uno strumento polifonico tricalamo che fa parte della famiglia degli aerofoni. Sono considerate lo strumento più rappresentativo e più significativo della musica sarda. Per gli aspetti etnomusicologici ed etnocoreologici di questo strumento, che esulano dagli argomenti principali di questo articolo, rimando a Bentzon 2002 e a Lutzu 2012.

27. Intervista del 24/05/2017.

28. Va evidenziato che questa libertà espressiva è appannaggio quasi esclusivo degli uomini. Qui evidentemente subentra una questione relativa ai rapporti di genere nel ballo sardo, che non posso affrontare in questo scritto. Ma che, essendo una questione tanto ignorata quanto a mio avviso importante e poiché proprio per questo ho iniziato ad indagare, mi riprometto di trattarne in un futuro prossimo.

29. Intervista del 19/12/2013.

30. Ringrazio Mauro Frigau, allievo di Tonio Spiga, che mi ha aiutato a descrivere la peculiare struttura dei balli campidanesi, così come a interpretare e comprendere meglio lo stesso insegnamento del maestro.

31. Mutuo questa declinazione del capitale culturale di ascendenza bourdieusiana da Sarah Thornton che ha proposto il concetto di «capitale sottoculturale» in riferimento alle sottoculture giovanili dei club e dei rave (Thornton 1998). Avrei forse potuto continuare ad usare la denominazione di capitale sottoculturale, ma il diverso campo di applicazione mi ha fatto optare per la denominazione di capitale culturale subalterno, che peraltro ritengo ancora provvisoria.

32. A questo riguardo può essere utile notare come tra valenti ballerini e ballerine che hanno deciso di guidare dei corsi di ballo sardo alcuni non si ritengono propriamente insegnanti in quanto non possono avere un titolo ufficiale che riconosca tale status (ammettendo quindi implicitamente la subalternità culturale delle danze sarde). Altri invece fanno loro la denominazione di insegnante o di maestro/a poiché ritengono che di fatto quello è il loro ruolo (affermando in questo caso, anche qui implicitamente, che il ballo sardo è una forma espressiva codificata e complessa propria di un orizzonte culturale che, al momento, non ha la possibilità di farsi riconoscere ufficialmente dalle istituzioni egemoni: pertanto non rimane che l'autoinvestitura da parte dei ballerini più abili e intraprendenti, oltre il riconoscimento che proviene dagli allievi e, se c'è unanimità, dall'intera scena locale del ballo sardo).

33. Intervista del 19/12/2013.

34. Intervista del 24/05/2017.

35. Intervista del 19/12/2013.

36. Intervista del 24/05/2017.

37. Intervista del 28/10/2013. 


\section{RIASSUNTI}

Questo articolo si propone di mostrare alcune articolazioni poco esplorate della categoria di filologicamente corretto in ambito antropologico. La tesi che si intende dimostrare è che il filologicamente corretto non sia solo uno strumento critico al servizio della visione istituzionale e colta, o uno strumento retorico a disposizione delle politiche patrimoniali: può invece anche essere impiegato dal basso ai fini di una micro-contro-egemonia locale. La trattazione si svilupperà partendo da un inquadramento concettuale del filologicamente corretto e confrontandolo col concetto affine ma differente di autenticità. Verrà poi illustrata la nozione di campo del folklore come ciò che dà senso e ragione ai processi di autenticazione e alla pratica del filologicamente corretto in un contesto di cultura popolare riflessiva. Il focus etnografico sarà concentrato sulla particolare traiettoria del filologicamente corretto nel contesto della pratica delle danze sarde dette tradizionali, con particolare riguardo all'operato di un maestro di ballo sardo campidanese, che verrà considerato come un filologo popolare che si muove in una scena sfaccettata e polemica della quale fanno parte gruppi folklorici, cultori della danza e ballerini di piazza.

This article aims to show some little explored articulations of the philologically correct category in the anthropological field. The thesis that we intend to demonstrate is that the philologically correct is not only a critical tool at the service of institutional and cultured vision, or a rhetorical tool at the disposal of heritage policies: it can also be used from below for the purpose of a local micro-counter-hegemony. The dissertation will develop starting from a conceptual framework of the philologically correct and comparing it with the similar but different concept of authenticity. The notion of folklore field will then be illustrated as giving meaning and reason to the processes of authentication and to the practice of philologically correct in a context of reflexive popular culture. The ethnographic focus will be based on the particular trajectory of the philologically correct in the context of the practice of so called traditional Sardinian dances, with particular regard to the work of a Sardinian Campidanese dance master, who will be considered as a popular philologist moving in a multifaceted and polemic scene which includes folk troups, dance enthusiasts and dancers "in the field".

\section{INDICE}

Keywords : philologically correct, folklore field, dance, Sardinia, hegemony, subalternity Parole chiave : filologicamente corretto, campo del folklore, danza, Sardegna, egemonia, subalternità

\section{AUTORE}

\section{ALESSANDRO DEIANA}

Dipartimento di Scienze Sociali e delle Istituzioni, Università di Cagliari

alessandro.deiana803@gmail.com 